\title{
Adaptive Supporting Prioritized Soft Handoff Calls for Power-Controlled DS-CDMA Cellular Networks
}

\author{
Wen Chen ${ }^{1}$, Feiyu $\mathrm{Lei}^{1}$, Weinong Wang $^{1}$, and Xi Chen ${ }^{2}$ \\ 1 Network Information Center, Computer Science \& Engineering Dept., \\ Shanghai Jiao Tong University, 1954 Huashan Road, Shanghai 200030, China \\ wen.chenwen@gmail.com, \{fylei, wnwang $\}$ sjtu.edu.cn \\ 2 Computer Center, China ShipBuilding Industry Corporation, \\ No.701 Research and Development Institute, Wuhan Hubei 430070, China \\ cx040504@gmail.com
}

\begin{abstract}
We present feedback control techniques to intelligently support priorities of soft handoff calls during call admission control (CAC) in power-controlled DS-CDMA multicellular networks. We design a classic proportional controller to dynamically solve resource management problems, which arise during run-time adaptation, via continuously monitoring real-time system performance to adjust system parameters accordingly. Performance evaluation reveals that the solution not only has excellent stability behavior, but also meets zero steady state error and settling time requirements.
\end{abstract}

\section{Introduction}

It is well known that rejection of a handoff request causes forced termination of an ongoing service and wasting wireless resources due to retransmission. Therefore, the dropping of a handoff call is generally considered more serious than blocking of a new call. A basic approach to reduce handoff probability is to give handoff calls priority over new calls. In $2 \mathrm{G}$ TDMA/FDMA wireless networks, the popular guard channels (GC) [1] scheme and its numerous variants [2] exclusively reserve a fixed number of channels for handoff calls to make it. But the fundamental premise of the fixed GC schemes [3] is that the network behavior can be made to be deterministic through extensive a priori knowledge about network parameters. Therefore, they perform poorly in unpredictable dynamic systems.

Currently, emerging mobile wireless network such as DS-CDMA cellular networks are characterized by significant uncertainties in mobile user population and system resource state (i.e. soft capacity, soft handoff). Then, any solution for reducing handoff dropping in DS-CDMA systems must be highly adaptive for adherence to the desired system performance requirements, and cannot rely on the assumptions of traffic or mobility patterns. Feedback control theory can just be the theoretical basis for the design of adaptation-based architectures that handle QoS-aware services for current wireless networks with parametric, structural and environmental uncertainties. 
Although several recent studies [4] [5] have been conducted concerning the forced-termination of calls due to soft handoff failure in DS-CDMA networks, the adaptation mechanisms are not considered at all. And that some adaptive QoS schemes such as [6] realize the adaptive control only by a predefined fixed stepwise way, which cannot react to the system changes efficiently.

In this paper, we propose a radically different approach to adaptively reduce soft handoff failure probability in DS-CDMA cellular networks based on feedback control theory. The main contribution of this paper is that adaptive system performance optimization and feedback control techniques are combined for modeling the unpredictability of the environment, handling imprecise or incomplete knowledge, reacting to overload and unexpected failures, and achieving the required performance levels. Our contribution can be summarized as follows:

- Formulating the reducing handoff dropping problem as a feedback control loop. And we choose to use a P(Proportional) control function to adjust some system parameter adaptively according to the real-time change of network performance, not as previous stepwise way (such as [6]). And that we use the Root Locus method to tune the controller so as to satisfy the performance specs.

- Through comparing real-time soft handoff failure probability with new call blocking probability in the controller, we not only characterize real-time system performance variances accurately but also achieve a satisfied tradeoff between them.

- Using system identification to design a mathematical model that describes the dynamic behavior of CAC process in a cellular network.

- Achieving the desired network performance with traffic conditions and user mobility that are unknown a priori.

\section{Reference CDMA Cellular Network}

We consider a multicellular network with spread signal bandwidth of $\mathrm{WHz}$. We only focus on the uplink since it is generally accepted that it has inferior performance over the downlink.

\subsection{Traffic Classes}

We consider cellular networks that support both voice and data services. Assumed that there are $K(K \geq 1)$ different traffic classes with different QoS requirements. Namely, each class specifies their own transmission rate, activity factor, desired SIR requirement, and maximum power limit that can be received at the base station. We assume that traffic from the same service class has the same QoS requirements. We define a mapping $\sigma: Z^{+} \rightarrow\{1, \cdots, K\}$ to indicate that the $n$th connection is from the service class $\sigma(n)$, where $Z^{+}$denotes the set of nonnegative integers. Also, call requests are classified into soft handoff call and new call requests. In this paper, we give higher priority to soft handoff calls than new calls within the same class. 


\subsection{Uplink Capacity in Power Controlled Multicellular Networks}

As the uplink is more critical to total capacity than downlink [7], we consider only the uplink capacity of a reference cell in a multicellular DS-CDMA network. Let $N$ be the number of connections served by BS currently. The power received at the base station from the user (mobile station, MS) of the $n$th connection is denoted by $S_{n}, n=1, \cdots, N$. In an SIR-based power-controlled DS-CDMA network [8], the maximum received power at BS is determined by maximum power limit $H_{k}$ for connections from service class $k=\sigma(n)$, then

$$
0<S_{n} \leq H_{k}, \quad \forall n=\{1, \cdots, N\}
$$

The maximum power limits $H_{k}, k=1, \cdots, K$, principally depend on [8] the maximum power $p_{k}$ that can be transmitted by a MS of class- $k$ and the expected value of path loss for class- $k E_{k}[L]$ from the cell boundary to the base station. Then, we can choose $H_{k}=p_{k} E_{k}[L], k=1, \cdots, K$. In this paper, the cases when $S_{n}>H_{\sigma(n)}$ for some received call $n$, BS will reject the call since otherwise either some MS (mobile station) would be required to transmit more power than they can possibly do or the acceptance of the new call will severely damage the QoS of existing connections.

Let $\alpha_{k}$ be the activity factor of a Class- $k$ user, the bit-energy-to-interference ratio $E_{\mathrm{b}} / N_{\mathrm{O}}$ for the $n$th connection at the $\mathrm{BS}$ can be expressed in terms of the received power of various connections existing in the considered cell (intra-cell) and the surrounding cells (inter-cell) as [8]:

$$
\left(\frac{E_{\mathrm{b}}}{N_{\mathrm{O}}}\right)_{n}=\frac{S_{n} W}{R_{\sigma(n)}\left(\sum_{i=1, i \neq n}^{N} \alpha_{\sigma(i)} S_{i}+I_{n}^{\text {other }}+\eta_{n}\right)},
$$

where $S_{i}$ is the power level of the $i$ th connection received at the base station, $R_{\sigma(n)}$ is the data rate of service class $\sigma(n), I_{n}^{\text {other }}$ is the total interference from neighboring cells, $\eta_{n}$ is the background(or thermal) noise. In [8], it had been shown that the total interference from neighboring cells, $I_{n}^{\text {other }}$, can be reckoned by

$$
I_{n}^{\text {other }}=f \sum_{i=1, i \neq n}^{N} \alpha_{\sigma(i)} S_{i},
$$

where $f$ is called the inter-cell interference factor with a typical value of 0.55 8]. The value of $f$ may not always be constant and can be updated properly to reflect changes in traffic conditions and distributions.

The soft capacity of CDMA systems is limited by the level of multiaccess interference measured by the SIR. In general, since SIR drops and the probability of packet error increases as the number of users increases, it appears reasonable to maintain SIR above set thresholds $\gamma_{k}, k=1, \cdots, K$ by limiting the number of users. For example, the QoS requirement for voice users with a maximum bit error rate of $10^{-3}$ can be satisfied by the power control mechanism setting $\gamma$ at a required value of $7 d B[8]$. Then we must hold $\left(E_{\mathrm{b}} / N_{\mathrm{O}}\right)_{n} \geq \gamma_{\sigma(n)}$ for all current 
calls to maintain BER (bit error rate) below a certain limit as the following inequality:

$$
\frac{S_{n} W}{R_{\sigma(n)} \gamma_{\sigma(n)}} \geq(1+f) \sum_{i=0, i \neq n}^{N} \alpha_{\sigma(i)} S_{i}+\eta_{n}, \quad \forall n \in\{1, \cdots, N\} .
$$

\subsection{The Scheme for Assign Priority to Soft Handoff Requests}

In general, handoff calls are payed more attention than new calls and we have to give priority to handoff calls [4] [5] 8]. The main idea of the present approach is as follows [8], we can choose a fixed threshold $T_{k}<H_{k}, k=1, \cdots, K$, for new calls of class- $k$ to allow higher priority for handoff calls of class- $k$. Thus, BS would admit less new calls in the case $T_{k}<H_{k}$ than in the case $T_{k}=H_{k}$. The call admission policy is given as follows:

1. if a new call marked by connection- $(N+1)$ arrives, then

if $S_{N+1} \leq T_{\sigma(N+1)}$ and inequality (4) is satisfied $\forall n=\{1,2, \cdots, N, N+1\}$, accept the call;

otherwise, reject the call;

2. if a soft-handoff call marked by connection- $(N+1)$ arrives, then

if $S_{N+1} \leq H_{\sigma(N+1)}$ and inequality(4) is satisfied $\forall n=\{1,2, \cdots, N, N+1\}$, accept the soft-handoff call;

otherwise, reject the soft-handoff call.

In the above algorithm, new call thresholds $T_{k}, k=1, \cdots, K$ are key design parameters which effect the new call blocking probability and handoff failure probability at first hand. With highly variable conditions, any solution for reducing handoff dropping probability in a system must be highly adaptive. In our scheme, the main contribution is that we apply feedback control theory to adjust the thresholds $T_{k}$ adaptively, so as to achieve satisfactory network performance and response to the system's real-time dynamic. In the next section, we will introduce the development of the feedback controller.

\subsection{Network Performance Parameters}

In this paper, we basically consider two performance measures in each service class, i.e. new call blocking probability of class- $k P_{k, \mathrm{~b}}$ and soft handoff call failure probability of class- $k P_{k, \mathrm{~h}}$. According to [5] [9], we evaluate the network performance by grade of service (GoS), which is defined as

$$
G o S_{k}=P_{k, \mathrm{~b}}+\omega P_{k, \mathrm{~h}}, \quad \text { for } k=\{1, \cdots, K\},
$$

where $\omega$ is a weighting factor to put greater importance on soft handoff call dropping probability and is set to 10 in most work [5] 9. Since the system capacity depends on the QoS difference between the two performance measures, it has been shown [5] that for a given service class, the system capacity is maximal when the new call blocking probability is equal to the weighted soft handoff failure probability. And in every monitoring period, defined the time interval $\left[t_{m-1}, t_{m}\right]$ as the $m$ th monitoring period, we compute the two performance measures for each class. 


\section{Our Feedback Control Approach}

In the next-generation wireless network, the resource requirements and the arrival state of service requests occur over time, so it is even more difficult to model because none of them is known a priori. These problems call for mechanisms that can control them effectively, without depending on detailed insight into their internal structure or on precise models of their behavior. Whereas feedback control strategy can be applied for behavior optimization in unpredictable or poorly modelled environments.

In our feedback control architecture, we define a set of control related variable for each service class $k=1, \cdots, K$ in the following:

$\diamond$ Controlled Variable $\triangle P_{k}: \triangle P_{k}=\omega P_{k, \mathrm{~h}}-P_{k, \mathrm{~b}}$. It means network performance output, which is measured and controlled. This way can balance the two performance measures simply and efficiently.

$\diamond$ Set Point: 0. It represents that system capacity arrives maximal when the new call blocking probability is equal to the weighted soft handoff failure probability [5].

$\diamond$ Error $E_{k}: E_{k}=0-\triangle P_{k}=-\triangle P_{k}$. It shows the difference between the set point and the current value of the controlled variable.

$\diamond$ Manipulated Variable $\triangle T_{k}$ : It is the quantity of the new call power threshold that is adjusted by the controller.

And a feedback loop of our architecture [12] is 1) the system periodically monitors and compares the controlled variable to the set point to determine the error; 2 ) the controller computes the required control with the control function of the system based on the error; 3 ) the actuators changes the value of the manipulated variable to control the system.

Note that for each service class, there is an independent feedback loop with the identical design process. For convenience, we will only discuss the whole design process for some service class $k, k=1, \cdots, K$, in later development.

\section{Threshold Feedback Loop Design}

We utilize feedback control theory and methodology [10 to design an adaptive new call power threshold adjuster for each service class with proven performance guarantees. The corresponding design methodology includes

1. Choosing $\mathrm{P}$ control as the basic controller model for each class- $k$ to compute the change to the power threshold of class- $k \Delta T_{k}$;

2. Using system identification to design the open-loop system model;

3. Tuning the control parameters and meeting performance specs requirements of adaptive system with Root Locus methods; 


\subsection{P Controller}

We choose $\mathrm{P}$ control as the basic feedback control techniques in adaptive threshold adjustment for the following reasons [1] 12. The rationale for using a $\mathrm{P}$ controller instead of a more sophisticated Controller, such as PID (ProportionalIntegral-Derivative) Controller, is that the controlled system includes an integrator in the adjustment of the new call power threshold (see the following (8)) such that zero steady state error can be achieved without an I (Integral) term in the Controller. The D (Derivative) term is not appropriate for controlling real-time systems because Derivative control may amplify the noise in new call blocking probability and soft handoff failure probability due to random workloads [10.

A basic form $\mathrm{P}$ control formula for controlling the change of the new call power threshold for some service class- $k$ in our scheme is

$$
\triangle T_{k}(t)=C_{k, P} E_{k}(t)
$$

where $C_{k, P}$ is a tunable parameter (see Sect. 6). At each sampling instant $m, \mathrm{P}$ controller periodically monitors the difference between new call blocking probability and weighted soft handoff probability for each class, and computes the manipulated variable $\triangle T_{k}(m)$ with the following control formula:

$$
\triangle T_{k}(m)=-C_{k, P} \triangle P_{k}(m),
$$

If $\Delta T_{k}(m)>0$, the new call power threshold of class- $k$ should be increased. Otherwise, the new call power threshold of class- $k$ should be decreased. Namely,

$$
T_{k}(m)=T_{k}(m-1)+\triangle T_{k}(m) .
$$

In our work, the controller's tuning process needs to base on a linear model of the controlled system. It will be addressed in the following.

\subsection{The Open-Loop System Model}

As a basis for the analytical design of a controller, we must establish a dynamic model to describe the mathematical relationship between the input and the output of a system. Here, the input of the open-loop model is the change to the new call power threshold of class- $k \triangle T_{k}(m)$. The output of the model (i.e. the controlled variable) is the difference between the new call blocking probability and soft handoff probability of class- $k \triangle P_{k}(m)$. However, modeling computing systems with unknown dynamics has been a major barrier for applying feedback control in adaptive resource management of such system. As system identification methodology [1] provides a practical solution for solving such modeling problems, we utilize it to establish a linear model for the controlled system with differential or difference equations.

Model Structure. We observe that the output of an open-loop network model depends on previous input and outputs of the model. Then, the reference cellular 
network of some service class- $k$ is modelled as a $n$th order difference equation with some unknown parameters,

$$
\triangle P_{k}(m)=\sum_{j=1}^{n} a_{k, j} \triangle P_{k}(m-j)+\sum_{j=1}^{n} b_{k, j} \triangle T_{k}(m-j) .
$$

There are $2 n$ parameters $\left\{a_{k, j}, b_{k, j} \mid 1 \leq j \leq n\right\}$ that need to be decided in an $n$th order model of service class- $k$. Next, we will apply least squares estimator to solve the problem.

Least Squares Estimator. Least-squares estimator 13 can estimate unknown parameters by recursion formula, if only a system is modelled to be the following standard structure,

$$
y(m)=\Phi^{T}(m) \Theta(m)+e(m),
$$

where $\Phi^{T}(m)$ denotes the input-output observation vector, $\Theta(m)$ denotes the unknown parameters vector, $e(m)$ represents noise. White noise input has been commonly used for system identification [13. The estimator is invoked periodically at every sampling instant. At the $m^{\text {th }}$ sampling instant, according to the above (10), we define the vectors $\Phi_{k}(m)$ and $\Theta_{k}(m)$ for service class- $k$ :

$$
\begin{aligned}
& \Phi_{k}(m)=\left(\triangle P_{k}(m-1) \cdots \triangle P_{k}(m-n) \Delta T_{k}(m-1) \cdots \Delta T_{k}(m-n)\right)^{T}, \\
& \Theta_{k}(m)=\left(a_{k, 1} \cdots a_{k, n} b_{k, 1} \cdots b_{k, n}\right)^{T} .
\end{aligned}
$$

Let $\boldsymbol{R}(m)$ be a square matrix whose initial value is set to a diagonal matrix with the diagonal elements set to 10 . The recursion formulas of the estimator's equations for class- $k$ at sampling instant $m$ are [13]:

$$
\begin{aligned}
\gamma_{k}(m)= & {\left[1+\Phi_{k}^{T}(m) \boldsymbol{R}(m-1) \Phi_{k}(m)\right]^{-1} } \\
\Theta_{k}(m)= & \Theta_{k}(m-1)+\gamma_{k}(m) \boldsymbol{R}(m-1) \Phi_{k}(m)\left[\triangle P_{k}(m)-\right. \\
& \left.\Phi_{k}^{T}(m) \Theta_{k}(m-1)\right] \\
\boldsymbol{R}(m)= & \boldsymbol{R}(m-1)-\gamma_{k}(m) \boldsymbol{R}(m-1) \Phi_{k}(m) \Phi_{k}^{T}(m) \boldsymbol{R}(m-1) .
\end{aligned}
$$

At sampling instant $m$, we substitute the current estimates $\Theta_{k}(m)$ (reckoned by (12) ) into (9), the estimator "predicts" a value of the model output $\triangle \hat{P}_{k}(m)$. The estimate error is $\triangle P_{k}(m)-\triangle \hat{P}_{k}(m)$. The objective of the least squares estimator is iteratively update the parameter estimates at each sampling instant so as to minimize $\sum_{0 \leq i \leq m}\left(\triangle P_{k}(m)-\triangle \hat{P}_{k}(m)\right)^{2}$.

Our experimental results (Sect.5) establish a second order difference equation of class- $k$ to approximate the input-output relation of the dynamic open-loop model,

$\triangle P_{k}(m)=a_{k, 1} \triangle P_{k}(m-1)+a_{k, 2} \triangle P_{k}(m-2)+b_{k, 1} \triangle T_{k}(m-1)+b_{k, 2} \triangle T_{k}(m-2)$.

\subsection{The Closed-Loop Feedback Design}

In this section, we obtain the transfer function of the closed-loop system model to analyze the system dynamic. First, we convert the open-loop controlled system model for class- $k$ in (14) to a transfer function $G_{k, \mathrm{O}}(z)$ in $z$-domain: 


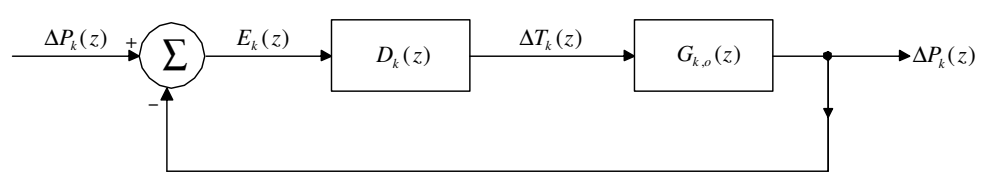

Fig. 1. New call power threshold of class- $k$ feedback control loop

$$
G_{k, \mathrm{O}}(z)=\frac{\triangle P_{k}(z)}{\triangle T_{k}(z)}=\frac{b_{k, 1} z+b_{k, 2}}{z^{2}-a_{k, 1} z-a_{k, 2}} .
$$

Second, the transfer function of the class- $k \mathrm{P}$ controller in $z$-domain is also given by

$$
D_{k}(z)=C_{k, P} .
$$

Thus, given the open-loop model and the controller model, we achieve the transfer function $G_{k, \mathrm{c}}(z)$ of the closed-loop model:

$$
G_{k, \mathrm{c}}(z)=\frac{D_{k}(z) G_{k, \mathrm{O}}(z)}{1+D_{k}(z) G_{k, \mathrm{O}}(z)}=\frac{C_{k, P}\left(b_{k, 1} z+b_{k, 2}\right)}{z^{2}+\left(b_{k, 1} C_{k, P}-a_{k, 1}\right) z+\left(b_{k, 2} C_{k, P}-a_{k, 2}\right)} .
$$

In summary, we present the block diagram of the adaptive new call power threshold of class- $k$ feedback control system in Fig 1 .

\subsection{Performance Specs}

To design adaptive systems, it is necessary to devise specifications for the adaptive process itself. The following metrics [10] of a closed-loop system are used to describe the quality of adaptation:

- Stability: BIBO (bounded-input bounded-output) stability, which means that the system output is always bounded for bounded references, is satisfied to avoid uncontrollable performance degradation in a system. In the context of our system, this means stability is a necessary condition to prevent the controlled variables $\triangle P_{1}$ from severe deviations with reference values 0 . For example, although $P_{1, \mathrm{~b}}$ and $P_{1, \mathrm{~h}}$ exceed some limit values to make the link availability low, the controlled variable $\triangle P_{1}$ unexpectedly reaches the set point. To satisfy the stability, it avoids the situation happening.

- Setting time $T_{s}: T_{s}$ is the time it takes the output to converge to within $2 \%$ of the reference and enter steady state. It represents the efficiency of the controller. We assume that our system requires the settling time $T_{s}<10$ sec.

- Steady state error: For a closed-loop system, the steady state error represents the accuracy of the basic controller in achieving the desired performance. And zero steady state error means our closed-loop controller can bring performance parameters to their set points in steady state with zero error. 


\section{System Identification Experiment}

We first conduct simulation studies for a network with single class of service (e.g., voice). And the value of threshold $T_{1}$ (assumed that $\mathrm{k}=1$ denotes voice class) changes in the range of $0.75 \cdot 10^{-14} \mathrm{~W}$ to $1.0 \cdot 10^{-14} \mathrm{~W}$ as the parameters used in [8]. We use the System Identification Toolbox of MATLAB to run the system identification experiments to respectively estimate a first order, a second order, a third order and a forth order model. Figure 2 demonstrates that the estimated first order model has larger prediction error than the second order model, while an estimated third/forth model does not tangibly improve the modeling accuracy. Hence the second order model is chosen as the best comprise between accuracy and complexity. The corresponding estimation parameters are $\left(a_{1,1}, a_{1,2}, b_{1,1}, b_{1,2}\right)=(0.6209,-0.06977,3.813 e 010,-2.645 e 007)$.

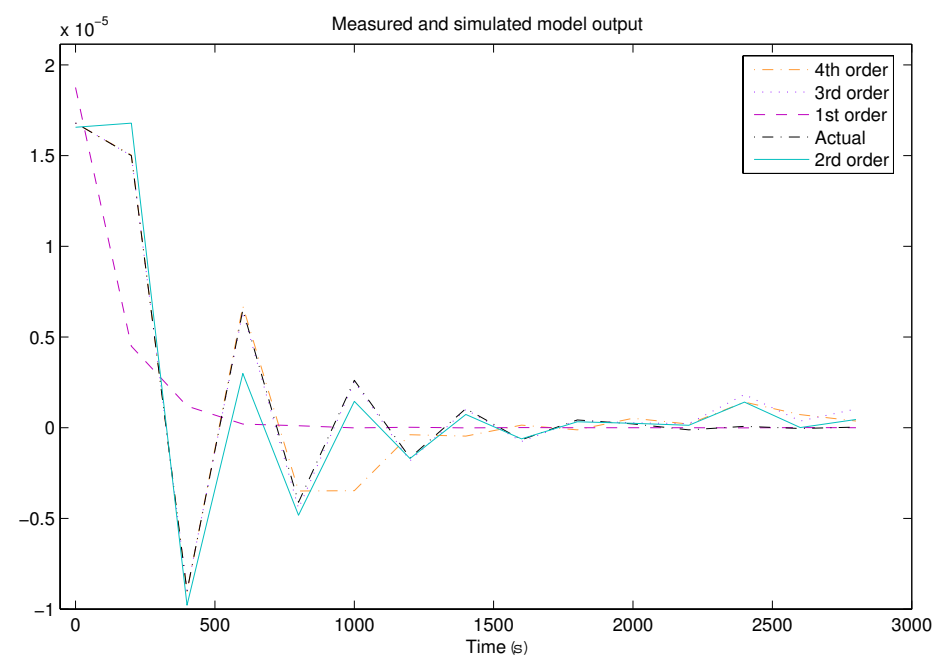

Fig. 2. System Identification Results of voice class

We can conduct estimation for cellular network with two or more than classes of services using the same way, for each class has a completely dependent controller and model with only relatively different network parameters (see [8]). Similarly, the following turning process of the controller for voice class also gives a demonstration.

\section{Control Tuning and Performance Analysis}

According to control theory, the performance profile of a system depends on the poles of its closed-loop transfer function. We can place the pole at the desired location by choosing the right vale for the control parameter $C_{1, P}$ (voice class) to achieve desired performance spec. 


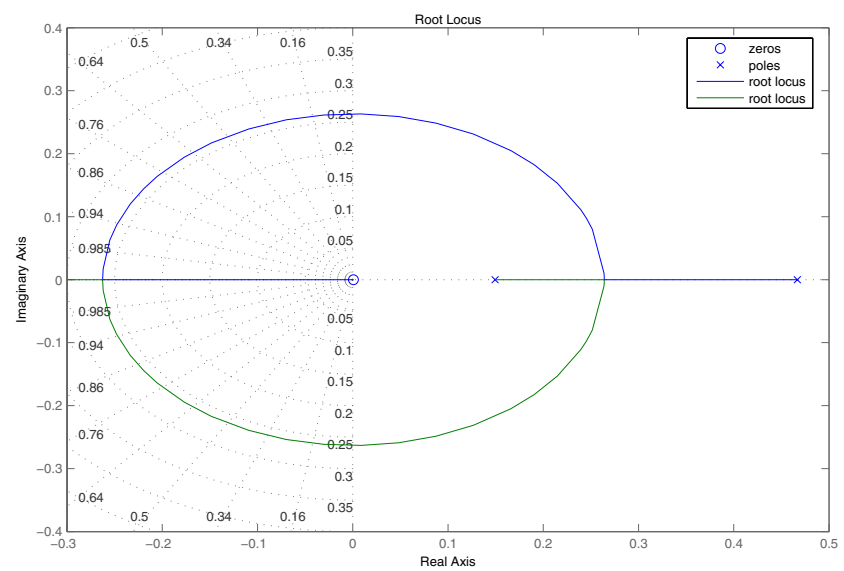

Fig. 3. Root Locus of the Closed-Loop Model

The Root Locus is a graphical technique that plots the traces of poles of a closed-loop system on the $z$-plane (or $s$-plane) as its controller parameters change. We use the Root Locus tool of MATLAB to tune the control parameter $C_{1, P}$ so that the performance specs can be satisfied. For the closed-loop model (17) (based on the estimated model parameters above), the traces of its closedloop poles are illustrated on the $z$-plane in Fig 3 . The closed-loop poles are placed at

$$
l_{1}=0.4721, \quad l_{2}=0.1478
$$

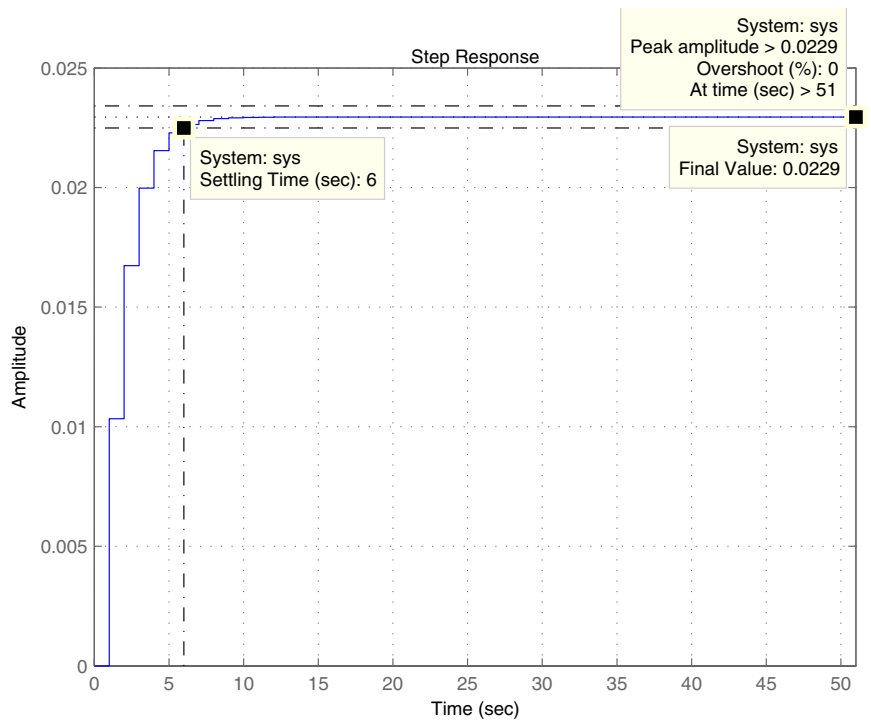

Fig. 4. Step Response of the Closed-Loop Model 
by setting the controller parameter to

$$
C_{1, P}=2.7097 e-014
$$

Hence, our closed-loop system obtains the following performance profile:

- Stability: The closed-loop system with the power threshold controller(based on the parameters in (19)) guarantees BIBO stability because the real roots of all the closed-loop poles are in the unit circle, i.e. $\left|l_{j}\right|<1(j=1,2)$ (see (18) and Fig 3).

- Setting time $T_{s}$ : From Fig 4 we observe that the controller achieves a settling time of $6 \mathrm{sec}$, lower than the required settling time $(10 \mathrm{sec})$.

- Steady state error: In our design, the controller achieves zero steady state error, i.e. $E_{s} \approx 0$ (see Fig 4). This means the closed-loop system can guarantee the desired performance in steady state.

In brief, the performance specs of our closed-loop system are proved to be satisfied. It demonstrates our adaptive architecture achieves robust QoS guarantee even when the environment varies considerably.

\section{Conclusion}

We have developed an novel adaptive new call power threshold adjustment algorithm based on feedback control theory. We have shown that the algorithm is stable and meets desired network performance. The algorithm is based both on a novel analytical model and employing standard feedback control design techniques using that model. This would be a new paradigm for adaptive QoS control in uncertain environments.

\section{References}

1. D. Hong and S.S. Rappaport. Traffic model and performance analysis for cellular mobile radio telephone systems with prioritized and no-protection handoff procedure. IEEE Transactions on Vehicular Technology, 35(3):77-92, 1986.

2. H. Chen, S. Kumar, and C.-C. Jay Kuo. Differentiated qos aware priority handoff in cell-based multimedia wireless network. IST/SPIE's 12th International Symposium, Electronic Imaging, January 2000.

3. Hossain Monir and Hassan Mahbub. Adaptive resource management in mobile wireless networks using feedback control theory. Telecommunication Systems, 25(34):401-415, 2004.

4. Wha Sook Jeon and Dong Geun Jeong. Call admission control for cdma mobile communications systems supporting multimedia services. IEEE transactions on wireless communications, 1(4):649-659, October 2002.

5. Jin Weon Chang and Dan Keun Sung. Adaptive channel reservation scheme for soft handoff in ds-cdma cellular systems. IEEE transactions on vehicular technology, 50(2):341-353, March 2001. 
6. Christoph Linderman, Marco Lohman, and Axel Thümmler. Adaptive call admission control for qos revenue optimization in cdma cellular networks. IEEE Transcations on vehicular technology, 10:457-472, 2004.

7. Mi-Sun Do, Youngjun Park, and Jai-Yong Lee. Channel assignment with qos guarantees for multiclass multicode cdma system. IEEE Transactions on Vehicular Technology, 51(5):935-948, September 2002.

8. Derong Liu, Yi Zhang, and Sanqing Hu. Call admission policies based on calculated power control setpoints in sir-based power-controlled ds-cdma cellular networks. Wireless Network, 10:473-483, 2004.

9. Sungmoon M. Shin, Cheol-Hye Cho, and Dan Keun Sung. Interference-based channel assignment for ds-cdma cellular systems. IEEE Transcations on vehicular technology, 48(1), January 1999.

10. Chenyang Lu, Tarek F. Abdelzaher, John A. Stankovic, and Sang Hyuk Son. A feedback control approach for guaranteeing relative delays in web servers. IEEE Real Time Technology and Applications Symposium, pages 51-62, 2001.

11. Gene F. Franklin, J. David Powell, and Abbas Emami-Naeini. Feedback control of dynamic systems (4rd ed.). 2004.

12. J. Stankovic, C. Lu, S. Son, and G. Tao. The case for feedback control real-time scheduling. EuroMicro Conference on Real-Time Systems, June 1999.

13. FK.J Astrom and Wittenmark B. Adaptive control (2nd ed.). 1995. 